\title{
Bio-functionalized (Ag-Ser) Nanoparticle Synthesis and Characterization for Biomedical Platforms
}

\author{
M. Saraçoğlu ${ }^{1}$, M. B. Bacınoğlu ${ }^{2}$, S. Mertdinç ${ }^{1}$, S. Timur ${ }^{1}$, B. T. Karaca ${ }^{3}$ \\ ${ }^{1}$ İstanbul Technical University, Material \& Metallurgy Department \\ Maslak, İstanbul, Turkey \\ saracoglum16@itu.edu.tr; mertdinc@itu.edu.tr; timur@itu.edu.tr \\ ${ }^{2}$ Isstanbul Technical University, Environmental Engineering Department \\ Maslak, İstanbul, Turkey \\ bacinoglu20@itu.edu.tr \\ ${ }^{3}$ Biruni University, Molecular Biology \& Genetics Department \\ Zeytinburnu, İstanbul, Turkey \\ btaktak@biruni.edu.tr
}

\section{Extended Abstract}

Several nanoparticle synthesis, characterization and functionalization methods are derived by selfassembly of peptides on Ag surface have been used for biomedical applications from drug delivery to biosensor systems [1]. Nanoparticle interaction with biological matrix is greatly affected by the morphology of the particle, surface chemistry, functionalization, and electronic and physicochemical properties. Understanding of biointerface science for nanoparticle and bio-matrix interaction mainly depends on particle size and surface characteristics [2].

Herein, a bio-functionalized nanoparticle synthesis route is designed and accomplished for biomedical applications. Sericin extracted from Bombyx mori silk cocoon is used for successfully controlled nucleation and further protection of silver nanoparticles. In the presence of sericin, silver amine complex $\left[\mathrm{Ag}\left(\mathrm{NH}_{3}\right)_{2}\right]+$ is reduced by sugar thus, stable silver particles are formed and maintain long term functionality such as 4 months at least. Newly synthesized nanoparticles are characterized by using various methods such as UV-visible spectroscopy, dynamic light scattering (DLS), transmission electron microscopy (TEM), and gel electrophoresis. Assembled nanoparticles, spherical in nature, have very narrow $(\sigma= \pm 5)$ size in distribution and peak at $20 \mathrm{~nm}$. The stability of particles has crucial importance to display functionality in a biological environment. Therefore, measuring absorbance with UV-vis spectroscopy assesses the stability of nanoparticles at various $\mathrm{pH}$ and $\mathrm{NaCl}$ concentrations to understand agglomeration kinetics of Ag-Ser NPs. Furthermore, critical agglomeration concentration is determined by agarose gel electrophoresis. Subsequently, surface charge of bio-functionalized Ag-Ser NPs is measured by Zeta-potential analyzer. The stabilization mechanism of bio-functionalized Ag-Ser NPs is analyzed by Raman spectroscopy to elucidate the main function of sericin on the synthesis process and molecular weight of extracted sericin is determined by sodium dodecyl sulfate-polyacrylamide gel electrophoresis (SDS-PAGE).

In order to understand biological efficiency of bio-functionalized Ag-Ser NP on pathogenic microorganisms, the minimum inhibitory concentration assay is carried out and the results indicates that around $3.38 \mu \mathrm{g} / \mathrm{ml} \mathrm{of}$ sericin-silver nanoparticle effectively inhibits the growth of Escherichia coli, Staphylococcus aureus, Pseudomonas aureginosa, Candida albicans. Comprehensively, the sericin-modified chemical reduction of silver nanoparticles offers easy, non-toxic nanoparticle synthesis with a short reaction time. With this method, the applicability of silver nanoparticles with high stability widens in many biotechnological research fields such as medical, electronics and photonics [3]. 


\section{References}

[1] Hnilova, M., Karaca, B. T., Park, J., Jia, C., \& Wilson, B. R. (2012). Fabrication of Hierarchical Hybrid Structures Using. Biotechnology and Bioengineering, 109(5), 1120-1130. https://doi.org/10.1002/bit.24405

[2] Auría-Soro, C., Nesma, T., Juanes-Velasco, P., Landeira-Viñuela, A., Fidalgo-Gomez, H., AcebesFernandez, V., ... Fuentes, M. (2019). Interactions of nanoparticles and biosystems: Microenvironment of nanoparticles and biomolecules in nanomedicine. Nanomaterials, 9(10). https://doi.org/10.3390/nano9101365

[3] Nel, A. E., Mädler, L., Velegol, D., Xia, T., Hoek, E. M. V., Somasundaran, P., ... Thompson, M. (2009). Understanding biophysicochemical interactions at the nano-bio interface. Nature Materials, 8(7), 543-557. https://doi.org/10.1038/nmat2442 